\title{
The Unusual Morphology, Structure, and Magnetic Property Evolution of Glassy Carbon upon High Pressure Treatment
}

\author{
C.Q. Jin, X. Wang, Z.X. Liu, Y.L. Zhang, F.Y. Li, and R.C. Yu \\ Institute of Physics, Beijing High Pressure Research Center, Chinese Academy of Sciences, P. O. Box 603, Beijing 100080, P. R. China
}

Received on 23 May, 2003.

\begin{abstract}
Glassy carbon (GC) has been high-pressure high-temperature treated. An interesting morphology evolution from the pristine sample to the high pressure products was observed. It is found that GC can be graphitized under pressure at a temperature much lower than that at ambient condition. Furthermore the $i n-s i t u$ structure and electrical measurements of GC and graphitized glassy carbon (GGC) under high temperature and high pressure have been investigated up to $30 \mathrm{GPa}$. We particularly emphasize the unusual magnetic properties of GC treated under high pressures and high temperatures. A paramagnetic to ferromagnetic-like, and then to superconducting (a diamagnetic signal with hysteresis magnetic response) -like behavior, which can be observed at temperatures as high as $80 \mathrm{~K}$, appears as a successive evolution from the initial GC to GGC in accordance with three regions distinguished by the graphitization temperature. This interesting evolution of magnetic properties probably evokes the new understanding of carbon element.
\end{abstract}

\section{Introduction}

It is fascinating to investigate the unusual superconductivity in materials containing only light element simply due to their relatively higher Debye temperature ${ }^{[1]}$. Carbon is one of such legendary element due to its many forms in terms of structure configuration ${ }^{[2]}$. Recent experiments reveal that even in pure highly oriented pyrolytic graphite (HOPG), ferromagnetism and superconductivity fluctuations can exist ${ }^{[3]}$. Many research works have revealed the superconductivity in the doped new form of carbon $\mathrm{C}_{60}{ }^{[4]}$. Moreover, the recent discoveries of superconductivity ${ }^{[5]}$ in graphite composite materials have triggered renewed investigation on carbonbased materials ${ }^{[6]}$. The results imply that the structure dimension or the topology may play some fundamental role in figuring out the physical properties of graphite related materials.

Carbon has a great diversity of structural and electronic properties depending on its many forms. The single, double, triple carbon-carbon bonds, and their conjugated forms lead not only to the well-known crystalline phases of graphite and diamond, but also to the large variations in structure as a function of temperature and to metastable disordered solids. As an important form in carbon family, glassy carbon is generally believed to contain approximately $100 \% s p^{2}$ valence bond, which has the short-range order of a strained graphitelike layer ${ }^{[7]}$. It is believed that the glass carbon with a turbostratic structure featured by randomly stacked graphite layers is intermediate between amorphous and the graphite form. It thus offers the opportunity to study the topology related effect on graphite related material. High pressure plays a unique role in designing, modifying, tailoring the struc- ture, morphology and consequently the physical properties of matter, such as those established in the research on high $T_{C}$ superconductors ${ }^{[8]}$. These can be further verified by a series of more recently discovered superconductivity upon the application of high pressure in such unusual elements like oxygen, lithium, boron, etc.

Since the effect of order-disorder developed in the high pressures and high temperatures polymerization process can result in topological defects in this covalently bonded material and cause the occurrence of unpaired spin, it is thus interesting to check what happened for this evolution, which can provide evidences to testify the appearance of magnetic properties induced by structural instability. These topological defects may still retain after graphitization and increase the hybridization of local charge density in graphite sheets, as well as lead to the superconductivity.

Here we report and summarize our recent work ${ }^{[9,10,11]}$ on the investigations of high pressure high temperature treated glassy carbon which exhibited some unusual physical properties such as the evolution from ferromagnetic-like to superconducting-like behavior appeared in accord with three regions distinguished by the graphitization temperature.

\section{Experimental}

All of the glassy carbon used for this work was commercial product (Alfa Aesar, 99.9\%). They were spherical powder of a size ranging from 20 to $50 \mu \mathrm{m}$. High pressure and high temperature treatments were carried out in a conventional cubic-anvil type high pressure equipment. The raw materials 
were carefully wrapped with Platinum foil to avoid contamination, and were put into BN tube. A graphite sleeve was used as an internal furnace with pyrophyllite as the pressure transmitting medium. The treating process was performed at 3.0 -5.0 GPa and $600{ }^{\circ} \mathrm{C}-1400{ }^{\circ} \mathrm{C}$ for averagely $15 \mathrm{~min}$, followed by quenching from high temperature and releasing of pressure. The samples were routinely characterized by $\mathrm{X}$-ray diffraction, SEM and Raman spectroscopy, etc.

The high pressure X-ray diffraction up to $31.6 \mathrm{GPa}$ and electrical measurements up to $23.6 \mathrm{GPa}$ of GC and GGC were carried out using diamond anvil cell (DAC). The Platinum powder was served as the inner pressure calibration standard in the synchrotron radiation experiment. The resistance measurements were made on an Intelligent Model ZL5 LCR Measurement System using the assembly as previously reported $^{[12]}$.

DC magnetization $M(H, T)$ measurements were performed on two magnetometers: Quantum Design MPMS5 and Oxford MagLab. Before measurements the instruments were carefully degaussed. In order to exclude the impurity effects, an ICP-AES method by IRIS/AP-ICP emission spectrometer was adopted to examine the traces of impurities. The total amount of $\mathrm{Fe}, \mathrm{Co}, \mathrm{Ni}$ was found less than $70 \mathrm{ppm}$ in the raw material examined in magnetic measurements.

\section{Results and Discussion}

\section{The High pressure graphitization of glass carbon}

The after-treated specimens have very interesting particle shape evolution from sphere to polyhedron and also the crystalline morphology and size development with increasing temperature at the fixed pressure, as shown in Fig. 1. This dynamic graphitization process can be clearly reflected on the X-ray diffractions, as shown in Fig. 2. GC has peaks corresponding to the $(h k)$ and (002) positions of graphite but the other $(h k l)$ peaks are not visible, which suggests that GC consists of strained graphite layers stacked in a disordered manner ${ }^{[13]}$. When treated under high temperatures and high pressures, GC probably has high internal stresses since its shape varied significantly. However, the effect of the growth of finite crystalline sizes would exceed that of the strain-induced broadening as temperature increases ${ }^{[14]}$. The structure slowly evolves toward the ideal graphite structure, and the enhanced crystal formation is indicated by the appearance of higher and sharp peaks. Fig. 3 gives the Raman spectra of GC treated under different temperatures at 5.0 $\mathrm{GPa}$. It has been found that the relative intensity of the 1360 $\mathrm{cm}^{-1}$ mode with respect to the $1580 \mathrm{~cm}^{-1}$ mode varies as the inverse of the crystal planer domain size ${ }^{[15]}$. As is evident from Fig. 3, Raman spectra show a distinct narrowing of the width of two bands. Such changes depend strongly on molecular structure, as the variations of its shape and size are clearly demonstrated in Fig. 1. As the molecular size and perfection in atomic order increase with graphitization, the intensity of the $1580 \mathrm{~cm}^{-1}$ mode increases, but the intensity of the $1360 \mathrm{~cm}^{-1}$ band decreases. When temperature reaches $1400^{\circ} \mathrm{C}$, a sudden structural change takes place in the sample, showing a typical Raman spectrum of polycrystalline graphite. This transformation temperature is much lower than the usual one with only heat treatment (normally around $\left.3000{ }^{\circ} \mathrm{C}\right)^{[16]}$ and at $1600{ }^{\circ} \mathrm{C}$ at $5 \mathrm{Kbar}^{[17]}$. This is simply due to the fact that the kinetics of transformation can be much accelerated under high pressure.

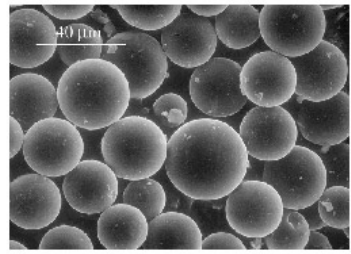

(a)

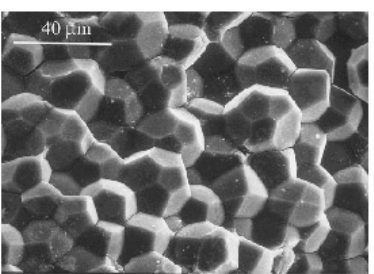

(c)

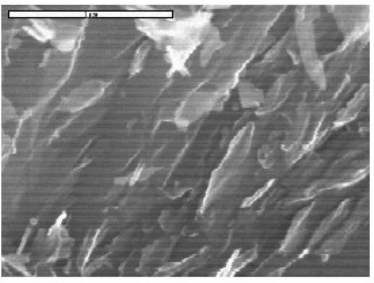

(e)

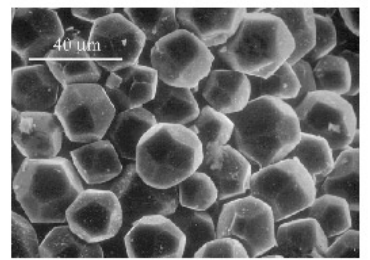

(b)

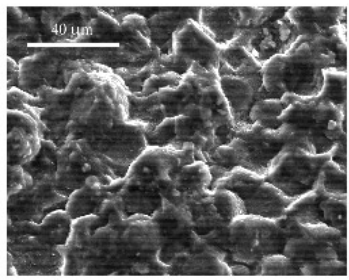

(d)

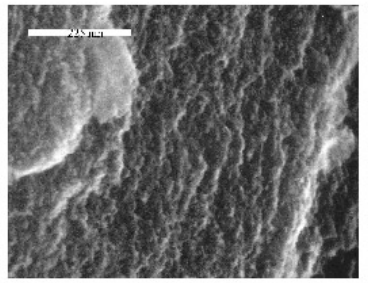

(f)
Figure 1. SEM photographs of GC prepared at 5.0 GPa, showing the particle shape evolution at (a) room temperature, (b) $600{ }^{\circ} \mathrm{C}$, (c) $1200{ }^{\circ} \mathrm{C}$, (d) $1400{ }^{\circ} \mathrm{C}$, and the crystalline morphology and size at (e) $1200{ }^{\circ} \mathrm{C}$, (f) $1400{ }^{\circ} \mathrm{C}$.

\section{High Pressure Effect on Structural and Electrical Prop- erties of Glassy Carbon}

The high pressure X-ray diffraction experiments for GC are carried out up to $31.6 \mathrm{GPa}$. Fig. 4 shows the energydispersive patterns for GC together with the inner calibrated element Pt. Fig. 4 shows the energy-dispersive patterns for GC together with the inner calibrate element Pt. The interplanar spacing $d_{002}$, basal plane dimension, $L_{a}$, and height $L_{c}$, normal to the (002) planes have been used extensively to characterize GC. The obtained $d_{002}$ spacing and the width at half maximum of the (002) band for GC and GGC are given in Fig. 5(a) and (b), respectively. From the pressure dependence of $d_{002}$-spacing in Fig. 5(a), GC has a larger $d_{002}$ 


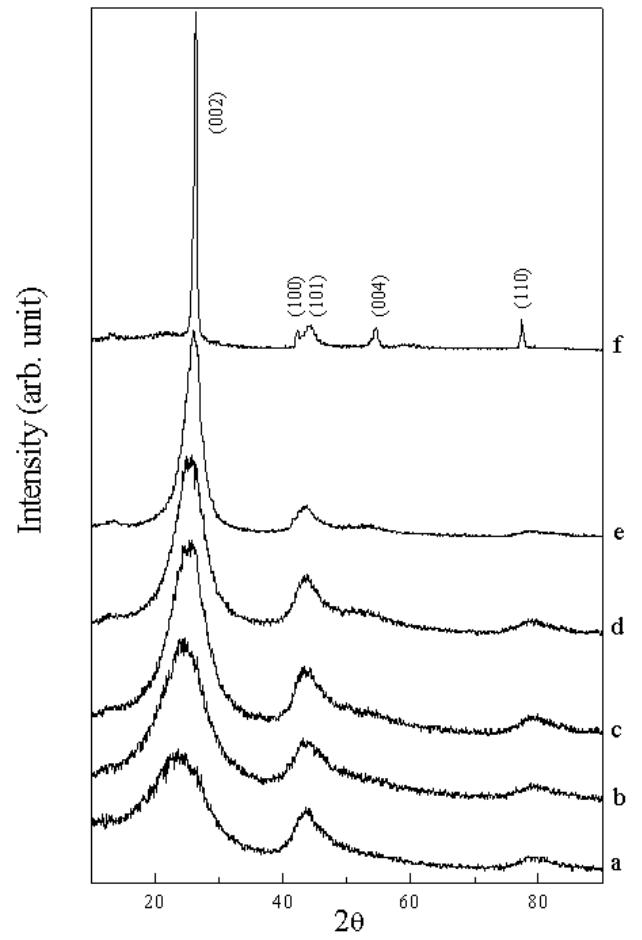

Figure 2. X-ray diffraction patterns of $\mathrm{GC}$ treated at $5.0 \mathrm{GPa}$ and at room temperature $(\mathrm{a}), 600^{\circ} \mathrm{C}(\mathrm{b}), 800^{\circ} \mathrm{C}(\mathrm{c}), 1000^{\circ} \mathrm{C}(\mathrm{d}), 1200^{\circ} \mathrm{C}(\mathrm{e})$, $1400^{\circ} \mathrm{C}(\mathrm{f})$.

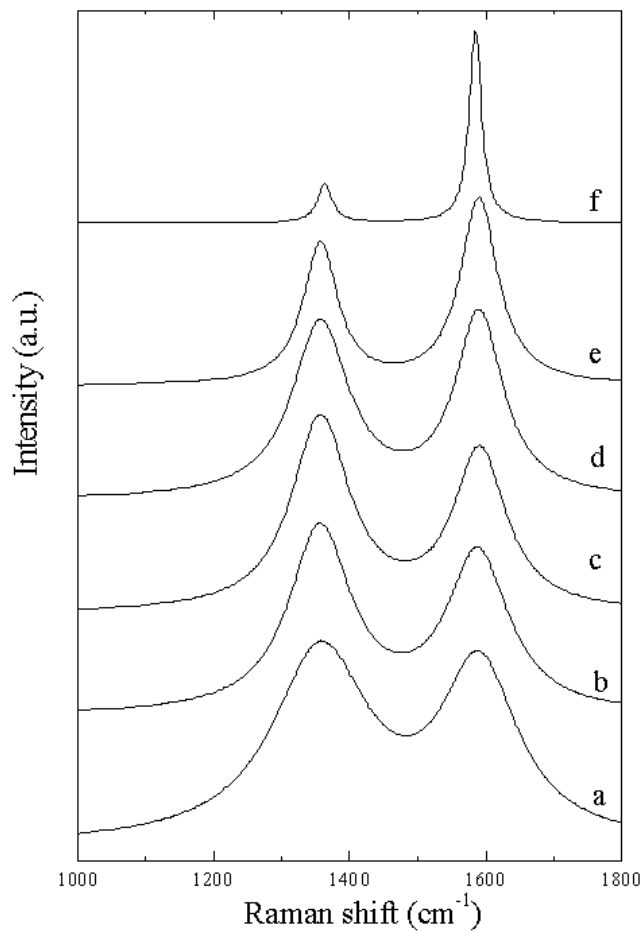

Figure 3. Raman spectra of the high pressure high temperature treated GC. (a) - (f) correspond to the same as of Fig. 2.

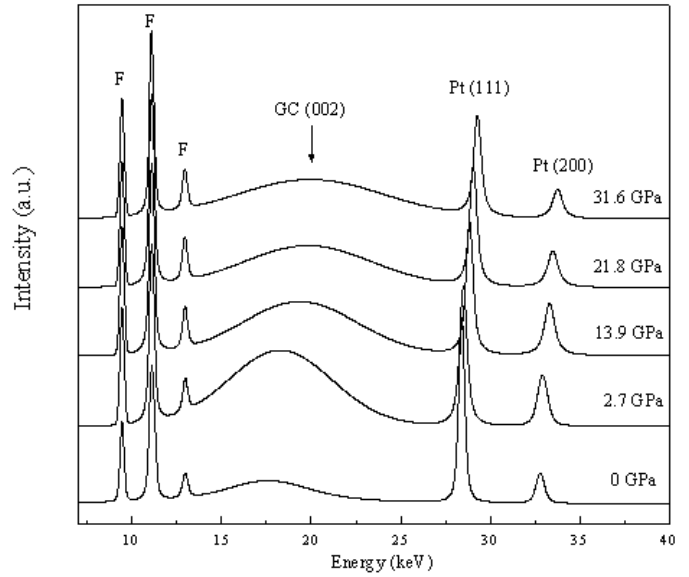

Figure 4. Energy-dispersive X-ray diffraction pattern of GC under various pressures.

than that of graphitized glassy carbon that has nearly the same $d_{002}$ as highly ordered pyrolitic graphite. The pressure dependence of the parameter $L_{c}$ derived from the Scherrertype modified Warren equation is also given in Fig. 5(b). With increasing pressure, the size of the graphitic layers and the apparent inter-layer spacing decrease continuously.

Bridgman presented a general formula to establish the equation of state (EOS) as: ${ }^{[18]}$

$$
\Delta V / V_{0}=a_{0}+a P+b P^{2}+c P^{3}+\ldots
$$

Where $\Delta V=V-V_{0}, V_{0}$ is the volume at zero pressure, with coefficients $a_{0}, a, b$, and $c$ being the fitting parameters, while the relative volume change $\Delta V / V_{0}$ can be directly referred from the relative density change. The density can be estimated from the X-ray diffraction data: ${ }^{[19]}$

$$
\delta_{X-\text { ray }}=\frac{Z A m_{H}}{a_{c}\left(a_{c} \sqrt{3} / 2\right) c}=\frac{7.627}{d_{002}} \mathrm{~g} / \mathrm{cm}^{3}
$$

where $Z=4$ denotes the number of carbon atoms in a unitcell, $A=12$ is the atomic weight of carbon, $m_{H}=$ $1.66 \times 10^{-24} \mathrm{~g}$ is the mass of a hydrogen atom, $a_{c}=2.456 \AA$ is the lattice constant of graphite. Fig. 5(C) shows a comparison of the $P-V$ relations of GC and GGC. It can be clearly seen that the change of $\Delta V / V_{0}$ of GC with increasing pressure is markedly different from that of GGC. The volume compression of GGC is much larger than that of GC indicating that a microstructural difference exists between them, which confirms the compressibility in carbon materials increases with increasing the degree of structural order.

The experimental $\Delta V / V_{0}-P$ data of GC and GGC have been fitted by the Bridgman equation and can be expressed as:

$$
\begin{gathered}
\Delta V / V_{0}=0.01207 P-0.00045 P^{2}+0.0000060027 P^{3}, \text { in GC; } \\
\Delta V / V_{0}=0.02637 P-0.00213 P^{2}+0.00009 P^{3}-0.0000012548 P^{4}, \text { in GGC. }
\end{gathered}
$$



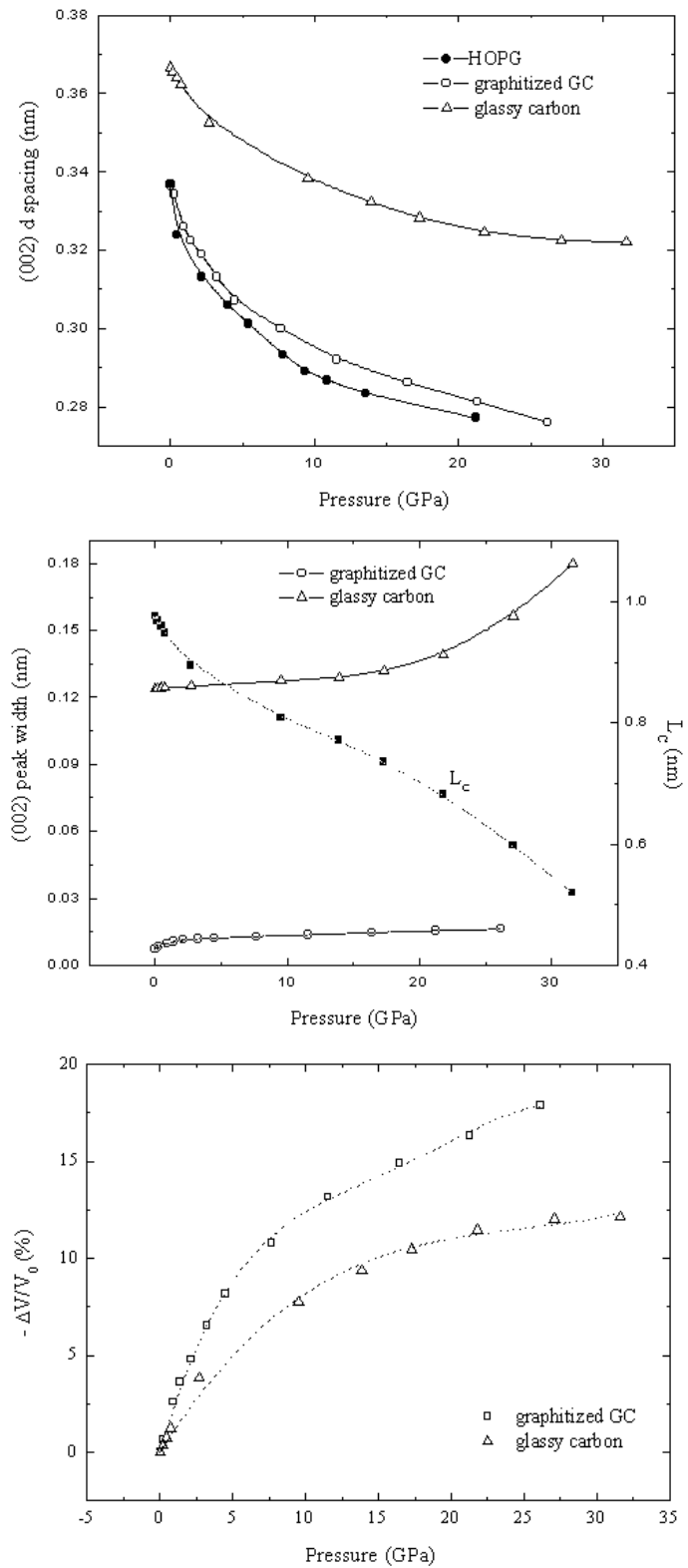

Figure 5. (a) The pressure dependence of $d_{002}$ for high-oriented graphite, GGC, and GC, (b) the effects of pressure on the peak width of $d_{002}$-spacing for GGC and GC, and $L_{c}$ for GC. (c) A comparison of the relative volume change $\Delta V / V_{0}$ between GGC and GC.

From the equation, we can obtain the bulk modulus $B_{0}$ according to the relationship, $B_{0}=1 / a_{0}$. The bulk modulus $B_{0}$ for GC and GGC is $82.9 \mathrm{GPa}$ and $38.0 \mathrm{GPa}$, respectively. The higher bulk modulus in GC indicates that it is much harder than GGC.

As can be seen from Fig. 6, both the resistances of GC and GGC decrease gradually with increasing pressure. At the low pressure for GGC, the unusual change of resistance in the curve indicates some rearrangement of packing of crystallites, or possibly some rearrangement of bonding at their edges when they are forced into contact. While in GC, the resistance decreases smoothly with increasing pressure up to about $13 \mathrm{GPa}$, then a bump appears in the $R$ - $P$ curve that indicates the completion of the merging between the orientated and disorientated layers. The evolution of free volume and reducing voids through compression in GC is expected to be sensitively related to the pressure relaxation. A short-range rearrangement of the atoms in graphite-like layers can be promoted by pressure. The difference of the scattering at boundaries between one graphite-like layer and the next is diminished with increasing pressure. On the other hand, the discontinuous graphite-like layers can cause small energy gaps between conduction band and valence band in the system, and applying an external pressure will decrease these gaps, and thereby increases the conductance in $\mathrm{GC}$, when the disorientated and orientated layers are totally merged.

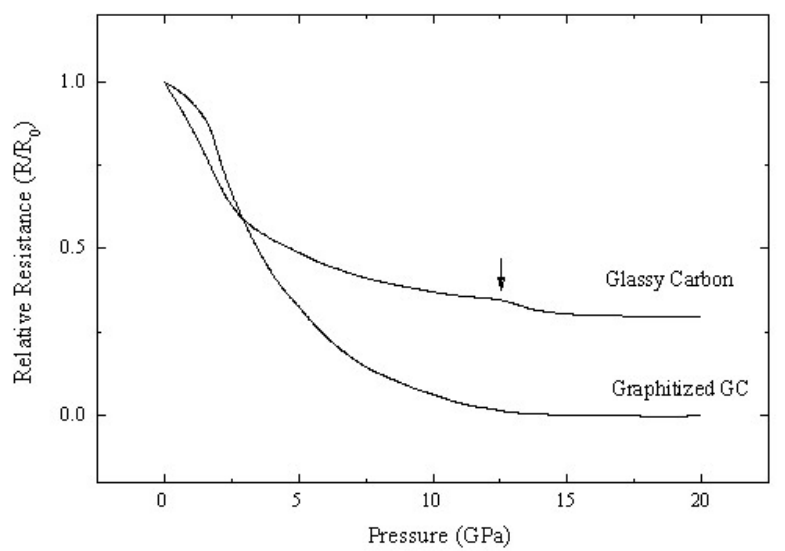

Figure 6. A comparison of the $R-P$ relation between GGC and GC. The arrow indicates a bump appearing in GC upon compression.

\section{The magnetic properties of the high pressure graphi- tized GC}

DC magnetization measurements have been performed for all products prepared under different pressures and temperatures. The temperatures in the high pressure synthesis have primary effects in distinguishing their magnetic behaviors. It can be divided into three regimes by the transformation temperature: the first one in non-graphitization region only shows paramagnetism, the second one in neargraphitization region reveals ferromagnetic signals, while the third one in graphitization region gives superconductinglike (a diamagnetic signal with hysteretic magnetic response) behaviors.

Figure 7 shows magnetization hysteresis loops measured in the field range between $\pm 1.6 \mathrm{kOe}$ at $10 \mathrm{~K}$ for glassy carbon prepared under different conditions. One is treated under $5 \mathrm{GPa}$ at room temperature, while the other is under the same pressure but at $1200{ }^{\circ} \mathrm{C}$ that is in the neargraphitization region. From this figure, one can clearly see that the sample at room temperature only reflects paramagnetic behavior while the sample at $1200{ }^{\circ} \mathrm{C}$ shows typical ferromagnetic hysteresis loop. It is not surprising for the appearance of paramagnetism since it is caused by local magnetic moments of dangling bonds, which exist extensively in amorphous carbon. With increasing synthesis temperatures, the products still show paramagnetic behavior until the 


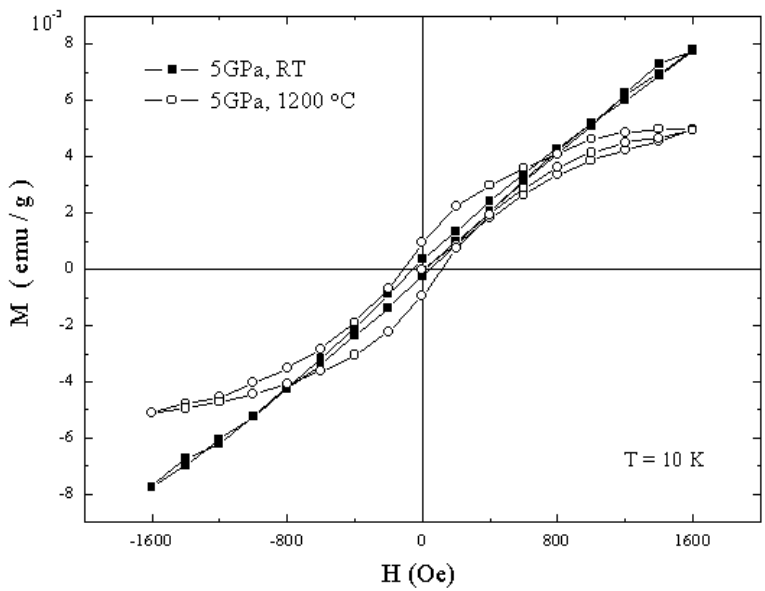

Figure 7. Magnetic moment hysteresis loops after subtraction of the diamagnetic background signals measured in samples treated at 5.0GPa at room temperature and $1200{ }^{\circ} \mathrm{C}$,respectively.

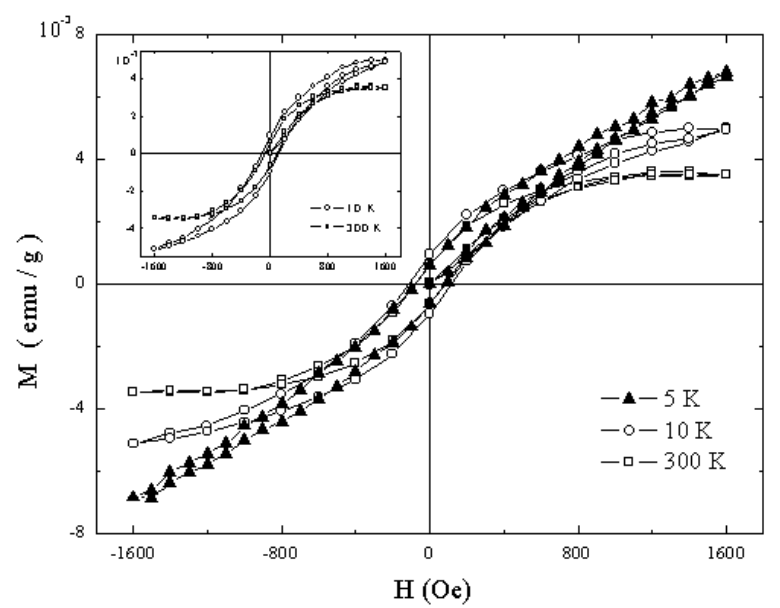

Figure 8. Magnetization loops for glassy carbon treated at $5 \mathrm{GPa}$, $1200{ }^{\circ} \mathrm{C}$ without subtracting background. The inset shows the results after subtracting the diamagnetic background data. It gives clear evidence for saturated magnetization at $300 \mathrm{~K}$.

temperature was close to the transition point $1400{ }^{\circ} \mathrm{C}$. Then a large hysteresis loop appears, as shown in Fig. 8 for $T=$ $5 \mathrm{~K}, 10 \mathrm{~K}$, and $300 \mathrm{~K}$ respectively. These hysteresis loops are typical for ferromagnets. A comparison between before and after (the inset) subtraction of the diamagnetic background signals is also presented. It has long been thought that ferromagnetism due to interactions between p-electrons is impossible. However, the discovery of a new organic material (TDAE- $\mathrm{C}_{60}$ ) rapidly changed this notion ${ }^{[20,21,22]}$. The ferromagnetic state can be a result solely of the alignment of p-electron spins on the fullerene units. From the point of this view, the observed unusual magnetic behavior is not too surprising because it may be the similar case for our samples treated under high temperatures and high pressures. The topological defects induced ferromagnetism is also used to explain the ferromagnetism in rhombohedral $\mathrm{C}_{60}{ }^{[23]}$. During the graphitization process, we have observed a significant shape change from spherical to polygonized. It can be clearly seen in Fig. 1(c), (e) that the topographical micrograph for the sample in near-graphitization region is of short-range order of the strained layers. We suppose that these shape changes are intimately related with the appearance of ferromagnetic behavior. These aggregated polyhedra with the short-range order of the strained graphite layer can lead to a $s p^{2}-s p^{3}$ rehybridization and form unpaired electron. This pressure-induced polygonization can trigger an ordering alignment of the itinerant moments coming from these unpaired spins to form itinerant ferromagnetism. When the prepared temperature reaches transition point, GC will transform into polycrystalline graphite. We also examine its magnetic signals and want to check if ferromagnetism still exists, but find that an interesting change from ferromagnetic-like to superconducting-like behavior happens. Shown in Fig. 9 are magnetic moment hysteresis loops $m(H)$ measured for $T=5 \mathrm{~K}, 40 \mathrm{~K}$ and $80 \mathrm{~K}$, respectively. One can clearly observe the superposition of the superconducting hysteresis loop over a linear diamagnetic background $\left(m_{0}=\chi H\right.$, where $\chi=-1.22 \times 10^{-6} \mathrm{emu}$ $\mathrm{g}^{-1} \mathrm{Oe}^{-1}$ at $T=10 \mathrm{~K}$ ). Measuring under higher temperatures will cause the detected signals comparable to background noise. Fig. 10 shows the temperature dependence of the magnetic moment $m(T)$ for applied field $H=100$ Oe. As shown in Fig. 10(a) for the sample in near-graphitization region, note the upturn around $50 \mathrm{~K}$, this can be attributed to the couples of paramagnetism of common amorphous carbon with some ferromagnetic clusters formed by defects as discussed above. While in Fig. 10(b), the sample belongs to polycrystalline graphite transformed by GC. Although Xray diffraction and Raman spectra have not shown any sign of amorphous carbon, we can not exclude the magnetic effects caused by them. The turning point seems at $T=20$ $\mathrm{K}$, but the magnetization reaches zero around $50 \mathrm{~K}$. It may indicate the existence of some untransformed GC. Our observed result has the similarity with the recent report about the existence of superconducting fluctuations in HOPG ${ }^{[3]}$. We speculate that the topological disorders have played an important role in the samples. The after-transformed glassy carbon is graphite microcrystalline of warped layers with curved regions, which can present some disclinations contributing to the disorder, as shown in Fig. 1(f). Localization due to this disorder can happen and enhance the local charge density, and therefore trigger the superconductivity. Since both the ferromagnetism and superconductivity in this material can be ascribed to the structural instability, during the process of graphitization they can coexist.

So far we have investigated the morphology, structure as well as magnetic response in the glassy carbon synthesized under different temperatures and pressures. The observed continuous occurrence of paramagnetism, ferromagnetism and superconductivity (diamagnetic like response) in accord with three regions distinguished by the graphitization temperature respectively would be reasonable if we take into account that the structural changes have a great impact on this magnetic behavior evolution. The dangling bonds in amorphous state are responsible for paramagnetism, and the itinerant spins may form in the polygonized glassy carbon, and after graphitization, the local disclinations can enhance the local charge density. Here we have proposed some spec- 
ulations about the observed experimental results. Fsurther studies are necessary to verify these hypotheses.

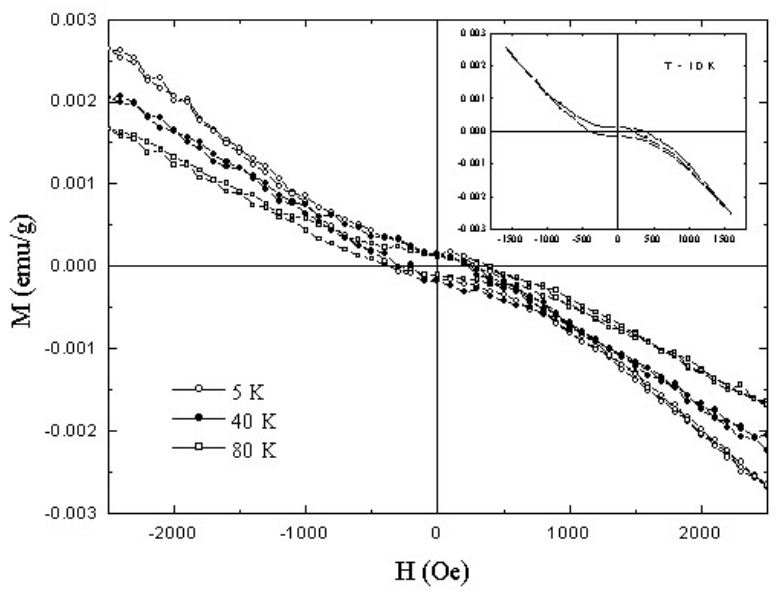

Figure 9. The superconducting-like magnetization behavior in GC after graphitization. The diamagnetic background signals have been subtracted. Continuing to increase temperature will cause the detected signals comparable to background noise.

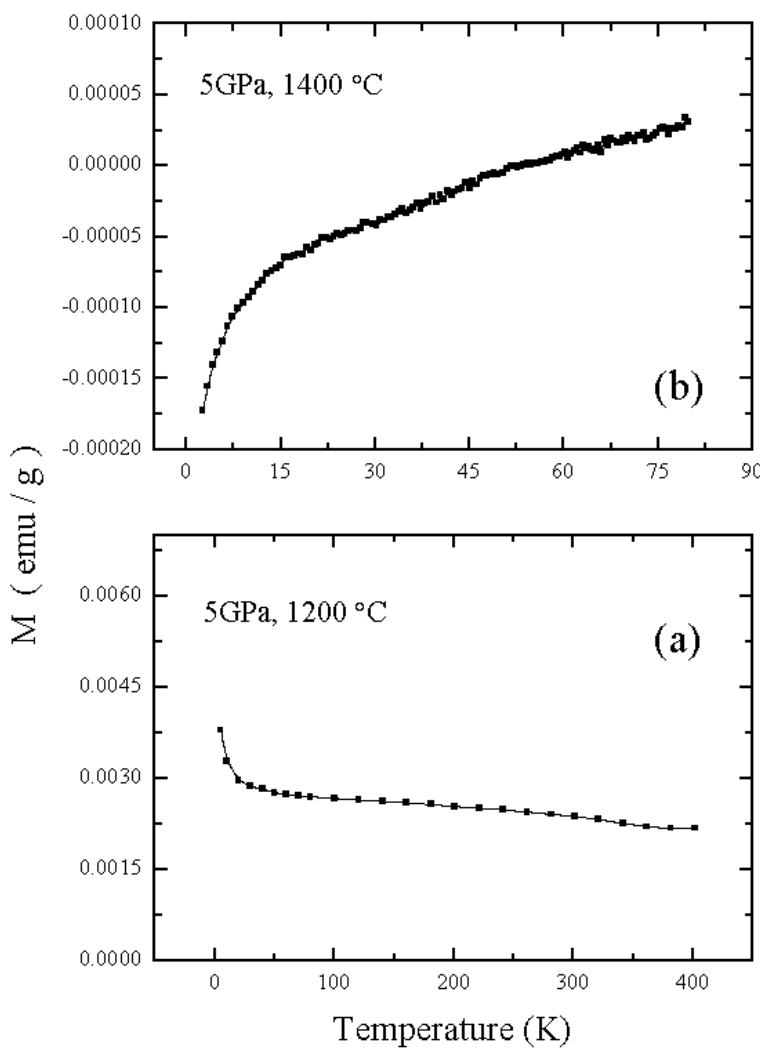

Figure 10. Temperature dependencies of the magnetic moment measured in glassy carbon before (a) and after (b) graphitization in applied magnetic field $H=100 \mathrm{Oe}$.

\section{Acknowledgement}

This work has been supported by the National Natural Science Foundation of China, Chinese Academy of Sci- ences, and the Ministry of Science and Technology of China.

\section{References}

[1] J. Bardeen, L. N. Cooper, and J. R. Schrieffer, Phys. Rev. 108, 1175 (1957).

[2] J. Robertson, Adv. Phys. 35, 317 (1986).

[3] Y. Kopelevich, P. Esquinazi, J.H.S. Torres, and S. Moehlecke, J. Low Temp. Phys. 119, 691 (2000).

[4] A.F. Hbard, M.J. Rosseinsky, R.C. Haddon, D.W. Murphy, S.J. Glarum, and T.T.M. Palstra, A.P. Ramirez, and A.R. Kortan, Nature 350, 600 (1991).

[5] R. Ricardo da Silva, J.H.S. Torres, and Y. Kopelevich, Phys. Rev. Lett. 87, 147001 (2001).

[6] J. Gonzälez, Phys. Rev. Lett. 88, 76403 (2002).

[7] G.M. Jenkins, K. Kawamura, and L.L. Ban, Proc. R. Soc. Lond. A. 327, 501 (1972).

[8] C. -Q. Jin, X. -J. Wu, P. Laffez, T. Tatsuki, T. Tamura, S. Adachi, H. Yamauchi, N. Koshizuka, and S. Tanaka, Nature 375, 301-303 (1995)

[9] X.Wang, Z.X.Liu, Y.L.Zhang, F.Y.Li, R.C.Yu, and C.Q.Jin, J. Phys.: Condens. Matter 14, 10265-10272 (2002).

[10] X.Wang Z.X.Bao, Y.L.Zhang, F.Y.Li, R.C.Yu, and C.Q.Jin, J. Appl. Phys. 93, 1991-1994 (2003).

[11] X. Wang, G.M. Zhang, Y.L. Zhang, F.Y. Li, R.C. Yu, and C.Q. Jin, Carbon 41, 188 (2003).

[12] Z. X. Bao, S. V. Hugo, and L. Francis Howell, J. Appl. Phys. 70, 6804 (1991).

[13] R.E. Franklin, Acta Cryst. 3, 107 (1950).

[14] R.R. Saxena, and R.H. Bragg, Carbon 16, 373 (1978).

[15] R.O. Dillon, J.A. Woollam, and V. Katkanant, Phys. Rev. B. 29, 3482 (1984).

[16] S. Yamada, and H. Sato, Nature 193, 261 (1962).

[17] M. Inagaki, A. Oberlin, and S. de Fonton, High Temp. - High Pressures 9, 453 (1977).

[18] P. W. Bridgman, The Physics of High Pressure (Bell and Sons, London, 1958).

[19] M. M. Dubinin, G. M. Plavnik, and E. D. Zaverina, Carbon 2, 261 (1964).

[20] P.M. Allemand, K.C. Khemani, A. Koch, F. Wudl, K. Holczer, S. Donovan, G. Gruner, and J.D. Thompson, Science 301, 253 (1991).

[21] A. Mrzel, A. Omerzu, P. Umek, D. Mihailovic, Z. Jagličić, and Z. Trontelj, Chem. Phys. Lett. 298, 329 (1998).

[22] B. Narymbetov, A. Omerzu, V. V. Kabanov, M. Tokumoto, H. Kobayashi, and D. Mihailovic, Nature 407, 883 (2000).

[23] T.L. Makarova, B. Sundquist, R. Höhne, P. Esquinazi, Y. Kopelevich, P. Scharff, V.A. Davydov, L.S. Kashevarova, and A.V. Rakhmanina, Nature 413, 716 (2001). 